\title{
Efficient Onboard Quantization for Multi-Channel SAR Systems
}

\author{
Michele Martone, Michelangelo Villano, Member, IEEE, \\ Marwan Younis, Senior Member, IEEE, and Gerhard Krieger, Fellow, IEEE
}

\begin{abstract}
In this letter, a novel method for onboard data reduction for multi-channel synthetic aperture radar (SAR) systems is presented. Such systems allow for high-resolution imaging of a wide swath but, on the other hand, require for their operation the acquisition and downlink of a huge amount of data: together with the intrinsic requirement related to resolution and swath width, this is due to the use of a pulse repetition frequency (PRF) typically higher than the processed Doppler bandwidth, which introduces a certain oversampling in the azimuth raw data. In this context, we propose a convenient data reduction strategy, named multi-channel block-adaptive quantization (MC-BAQ), which exploits the existing correlation between subsequent azimuth samples by performing a discrete Fourier transform of the multi-channel SAR data block. Then, a variable-bit quantization is applied, which allows for the optimization of the resulting performance and data rate. Simulations have been carried out on scenes with distributed scatterers showing different backscatter characteristics to demonstrate that the proposed MC-BAQ allows for a significant reduction of the data volume to be downlinked to the ground at the cost of a modest increase of onboard computational effort.
\end{abstract}

Index Terms-Multi-channel synthetic aperture radar (MCSAR), block-adaptive quantization (BAQ), data reduction, transform coding.

\section{INTRODUCTION}

$\mathbf{F}$ OR conventional single-channel SAR systems, it is well known that the pulse repetition frequency (PRF) poses opposite constraints for the imaging of wide swaths and, at the same time, of fine azimuth resolutions. Indeed, the former dictates a low PRF to allow for a sufficient temporal separation between subsequent SAR pulses, whereas the latter requires a large Doppler bandwidth and, therefore, high PRFs. Such inherent limitations can be overcome by exploiting multiple receiving apertures which are mutually displaced in alongtrack. The coherent combination of the individual received signals allows for adequate suppression of the ambiguous parts of the Doppler spectra and, in this way, high-resolution wideswath imaging is achieved [1], [2]. The downside for such an improvement of swath coverage and resolution, besides the increased system complexity, is represented by a significantly larger data volume to be acquired and transmitted to the ground, which poses more stringent demands on onboard memory and downlink capacity. In this scenario, efficient data volume reduction is of utmost importance, as the data rate selected for the digitization of the recorded radar signals

The authors are with the Microwaves and Radar Institute, German Aerospace Center (DLR), 82234 Wessling, Germany. e-mail: Michele.Martone@dlr.de directly affects the quality of the resulting SAR products. In the context of single-channel staggered SAR, a method for data volume reduction based on onboard Doppler filtering (i.e. exploiting the lower processed Doppler bandwidth with respect to the PRF) and decimation is proposed in [3]. However, this technique cannot be directly applied to the multi-channel case since the data of the individual receive channels are highly ambiguous. In the last years, a data-driven data reduction technique for multi-channel SAR based on a principal component decomposition has been proposed in [4], at the cost of a large onboard implementation effort. The approach we suggest in this letter aims at exploiting the intrinsic correlation among the azimuth samples. In particular, the multi-channel SAR data are decomposed by means of a nonadaptive orthogonal transformation (a simple discrete Fourier transform). Then, an optimized allocation of the quantization rates is applied to the transformed coefficients, which allows for an increase of the resulting performance for a preselected data rate. For data digitization, a standard block-adaptive quantizer (BAQ) is considered. The proposed method has been first introduced in [5], whereas in this letter a quantitative assessment in terms of achievable data volume reduction is presented, and the optimization of the bit allocation for the transformed coefficients is discussed in detail.

The letter is structured as follows: in the next section, multichannel block-adaptive quantization (MC-BAQ) for efficient data volume reduction is introduced. Section III presents simulations for the considered C-band single-platform multichannel SAR, which prove the effectiveness of the proposed compression scheme. The letter ends in Section IV with the conclusions and a brief outlook.

\section{Multi-Channel Block-Adaptive Quantization}

Let us consider a multi-channel SAR with $N$ receiving azimuth apertures. We define $v_{\text {sat }}$ as the satellite velocity, $\mathrm{PRF}_{\text {sys }}$ its transmit PRF, and $l$ as the azimuth separation between the sub-apertures, i.e. the total azimuth antenna length $L_{\mathrm{az}}=N \cdot l$. If the following constraint on the PRF is fulfilled

$$
\mathrm{PRF}_{\mathrm{sys}}=\frac{2 v_{\mathrm{sat}}}{L_{\mathrm{az}}},
$$

then the azimuth raw data stream is uniformly sampled, hence the resulting system is equivalent to a single-channel SAR with $\mathrm{PRF}_{\text {eff }}=N \cdot \mathrm{PRF}_{\text {sys }}$ and basically a conventional SAR processing can be applied. More in general, due to timing constraints and requirements on the ambiguity-to-signal ratio, the PRF is often selected so that (1) is not fulfilled. In this 


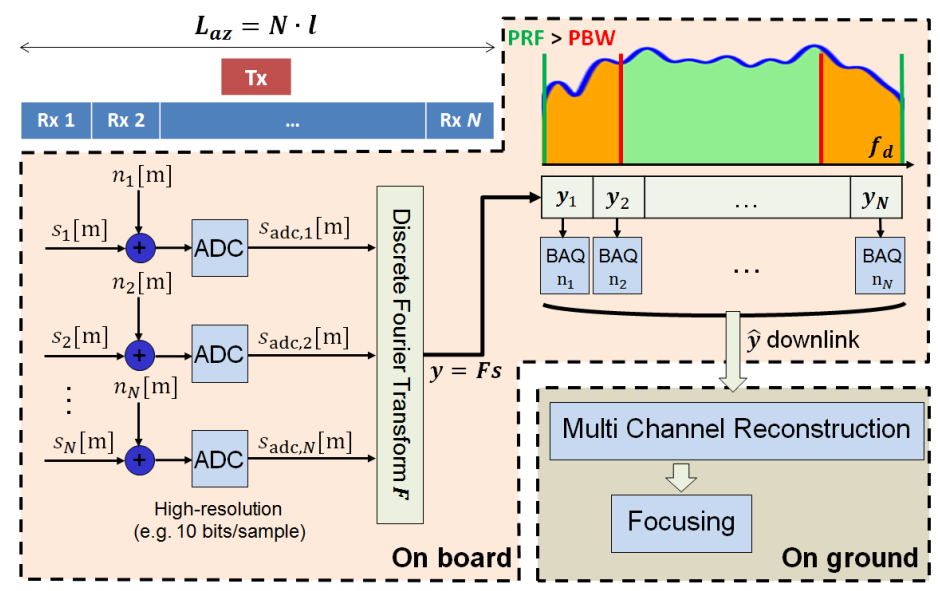

Fig. 1. MC-BAQ for onboard data reduction for a SAR system with multiple azimuth channels: for each time instant m, the signal received by the $i$-th azimuth channel, $s_{i}$ is first digitized by a high-precision analog-to-digital converter (e.g. 10-bit ADC). The multi-channel azimuth block of length $N$ is then decomposed by means of a discrete Fourier transform. As a next step, a proper bit allocation is applied to the transformed coefficients (the BAQ $\mathrm{n}_{i}$ blocks on the right-hand side) to optimize the resulting data volume and performance. The quantized coefficients $\hat{\boldsymbol{y}}$ are then downloaded to the ground, where inverse Fourier transform, multi-channel reconstruction, and SAR focusing are performed.

case, an appropriate signal reconstruction of the unambiguous Doppler spectrum of the non-uniform azimuth data, based on the generalized sampling expansion, needs to be carried out on ground by properly combining the $N$ subsampled channels as in [1], [6]. On the other hand, the Doppler bandwidth of the reconstructed multi-channel signal is larger than the system (transmit) PRF, i.e. a finer azimuth resolution is achieved with respect to the corresponding single-channel SAR operating with the full antenna length $L_{\mathrm{az}}$ and at the same $\mathrm{PRF}_{\text {sys }}$. Indeed, with multi-channel SAR systems a swath width of hundred kilometer and more can be imaged with an azimuth resolution in the order of one meter [2], [7]. For such a system, the raw data samples received by its $N$ azimuth channels exhibit a certain degree of correlation, which is introduced by the specific antenna pattern (or Doppler spectrum) together with a certain signal oversampling (defined by $\mathrm{PRF}_{\text {sys }}$ ) of the azimuth data stream [8]. Typically, for multi-channel SAR a processed Doppler bandwidth (PBW) that is significantly smaller than the product of the number of apertures and the PRF $\left(\mathrm{PBW}<N \cdot \mathrm{PRF}_{\text {sys }}\right)$ is required in order to get a sufficient azimuth ambiguity-to-signal ratio (AASR) [1], [2]. A direct downlink of the acquired multichannel data is, however, associated with an unnecessarily high data rate, as the effective PRF is significantly higher than the processed Doppler bandwidth. On the other hand, according to the specific system configuration, i.e., its antenna patterns, PRF, and processed bandwidth, one simple way to exploit the existing spectral selectivity is to perform a (lossless) discrete Fourier transform (DFT) on the multi-channel azimuth block before data compression. The DFT is chosen due to the intuitive correspondence between Doppler spectrum and antenna pattern, but in general other orthogonal transformations could be used for this purpose. Then, an efficient quantization strategy is implemented, which allocates less resources for those sub-bands which carry a smaller amount of information, i.e. that are located outside the processed bandwidth, and viceversa for those sub-bands lying in the more "useful" portion of the Doppler spectrum. By applying the transform coding paradigm, this strategy aims at removing the redundancy existing in the multi-channel data block and enables a better (more targeted) quantization, hence resulting in an increased quality of the final SAR image. For single-channel SAR, a similar compression scheme has been introduced in [9].

The workflow for the proposed onboard data reduction strategy for a multi-channel SAR with $N$ receiving azimuth apertures is sketched in Fig. 1: for each instant of time m, the signal received by the $i$-th azimuth channel $s_{i}\left(s \in \mathbb{R}^{N}\right)$ is first digitized by a high-resolution analog-to-digital converter. The multi-channel raw data block is then decomposed by the orthogonal transformation $\boldsymbol{F}$ into a set of $K$ azimuth beams $\boldsymbol{y}=\boldsymbol{F} \boldsymbol{s}(\boldsymbol{F}$ corresponds to the discrete Fourier transformation matrix and $\boldsymbol{y} \in \mathbb{R}^{K}$ ), each one corresponding to a different portion of the Doppler spectrum, and derived as

$$
y_{k}[\mathrm{~m}]=\sum_{i=1}^{N}\left(s_{\mathrm{ADC}, i}[\mathrm{~m}]\right) e^{-j 2 \pi i k / N} k=[1,2, \ldots, K] .
$$

In our case, without loss of generality, we assume the number of output coefficients $K$ to be equal to the number of the input samples, i.e. $K=N$ and $\boldsymbol{F} \in \mathbb{R}^{K \times N}$ (for $K<N$ the transformation is not strictly orthonormal). The output transformed coefficients are then further compressed by means of a set of block-adaptive quantizers (BAQ) [10], which are indicated on the right-hand side of Fig. 1. For this, a proper selection of each compression rate $\mathrm{n}_{k}$ associated to the $k$-th coefficient $y_{k}$ needs to be applied. The set of quantized coefficients $\hat{\boldsymbol{y}}$ is then downloaded to the ground, where the inverse transform, multichannel reconstruction, and SAR focusing are finally carried out. The optimum bit rate to be selected for the $k$-th output channel is derived from rate-distortion theory [11] as

$$
R_{k}=\bar{R}+\frac{1}{2} \log _{2} \frac{\sigma^{2}{ }_{k}}{\left[\prod_{l=1}^{K} \sigma_{l}^{2}\right]^{1 / K}}=\bar{R}+\Delta R_{k},
$$

where $\bar{R}$ is the mean allowed bit rate, $\sigma_{k}^{2}$ is the power associated to the $k$-th sub-band, and $\Delta R_{k}$ is the resulting 
TABLE I

MULTI-CHANNEL SAR SYSTEM PARAMETERS.

\begin{tabular}{|l|l|}
\hline Parameter & Value \\
\hline Satellite height, $h_{\text {sat }}$ & $700 \mathrm{~km}$ \\
\hline Carrier frequency, $f_{c}$ & $5.5 \mathrm{GHz}(\mathrm{C}$-band) \\
\hline Antenna type & Planar array \\
\hline Azimuth antenna length, $L_{\mathrm{az}}$ & $12.8 \mathrm{~m}$ \\
\hline Number of azimuth channels, $N$ & 8 \\
\hline Pulse repetition frequency, $\mathrm{PRF}$ & $1265 \mathrm{~Hz}$ \\
\hline Total processed bandwidth, $\mathrm{PBW}$ & $5630 \mathrm{~Hz}$ \\
\hline Target azimuth resolution, $\Delta_{\mathrm{az}}$ & $1.2 \mathrm{~m}$ \\
\hline Swath width, $W$ & $100 \mathrm{~km}$ \\
\hline ADC Resolution & $10 \mathrm{bits}$ \\
\hline
\end{tabular}

bit rate contribution to be added $\left(\Delta R_{k}>0\right)$ or subtracted $\left(\Delta R_{k}<0\right)$ for the $k$-th channel. Clearly, $\sum_{k=1}^{N} \Delta R_{k}=0$. It is worth pointing out that the above equation strictly holds for high rates (i.e. the pdf of the quantization error is approximately uniform in the decision interval), which may not be the case for the considered scenario. This aspect will be further discussed in the following. The power contribution $\sigma^{2}{ }_{k}$ is estimated as the fraction of the corresponding power spectrum integrated over the processed Doppler bandwidth as

$$
\sigma^{2}{ }_{k}=\frac{\mathbf{P}_{k}}{K^{2}} \int_{-\mathrm{PBW} / 2}^{\mathrm{PBW} / 2}\left|\frac{\sin \left(\pi K \frac{f+\mathrm{PRF}(K / 2-k) / K}{\mathrm{PRF}}\right)}{\sin \left(\pi \frac{f+\mathrm{PRF}(K / 2-k) / K}{\mathrm{PRF}}\right)}\right|^{2} d f .
$$

Hence, aside from the normalization factor $1 / K^{2}, \sigma^{2}{ }_{k}$ is proportional to the integral of the Dirichlet kernel of the discrete Fourier transform over the processed bandwidth PBW, scaled by the power associated to the $k$-th transformed coefficient $\mathbf{P}_{k}$. This latter term, in turn, represents the contribution of the antenna pattern in the corresponding portion of the Doppler spectrum, and can be numerically estimated by means of Monte-Carlo simulations for sequences of coefficients $y_{k}$ of sufficient length $L$ as

$$
\mathbf{P}_{k}=\frac{1}{L} \sum_{\mathrm{m}=1}^{L}\left|y_{k}[\mathrm{~m}]\right|^{2} .
$$

As a result from (3), $R_{k}$ is typically a non-integer number: fractional quantization rates can be implemented by toggling the bit rate selection of an integer-bit BAQ quantizer along azimuth and/or range, as already discussed in [12]. This way, higher flexibility of compression is achieved without increasing the overall scheme complexity.

\section{Simulation Results and Bit Rate Optimization}

In this letter we focus on a single-platform C-band multichannel SAR with $N=8$ azimuth channels and described in Table I. Fig. 2 shows the patterns for the transmit antenna (in blue) and a single receiver element (in red) for the considered system. Phase spoiling is applied in transmission [13], and a system $\mathrm{PRF}_{\text {sys }}$ of $1265 \mathrm{~Hz}$ is selected. The actual processed bandwidth PBW is represented by the shaded orange area and is of about $5.6 \mathrm{kHz}$, which corresponds to about $55 \%$ of the effective PRF (delimited by the dashed green lines). According to these system assumptions, the azimuth raw data stream is non-uniformly sampled (i.e. the relation in (1) is not fulfilled), hence a reconstruction of the unambiguous Doppler

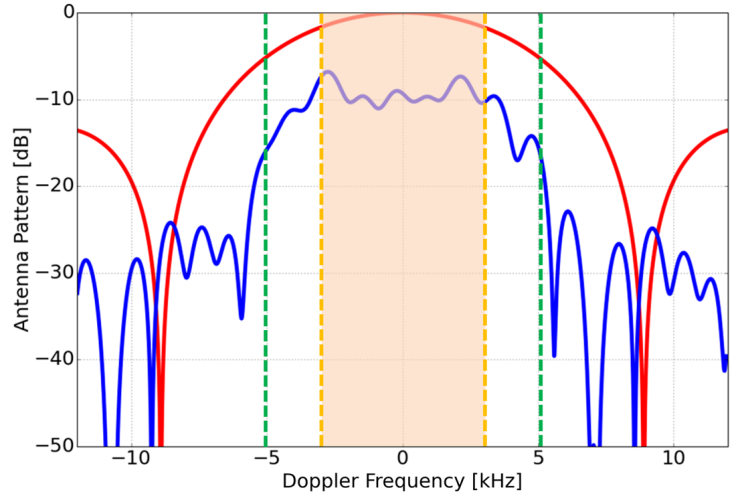

Fig. 2. Transmit (blue) and single element receive patterns (red) versus Doppler frequency (in transmission a phase spoiled pattern is employed). The shaded orange area indicates the processed bandwidth, whereas the effective sampling bandwidth $\left(N \cdot \mathrm{PRF}_{\text {sys }}\right)$ is delimited by the dashed green lines.

spectrum needs to be carried out on ground. According to, e.g., [1], this essentially consists of $N$ linear filters that are individually applied to the subsampled signals of the receive channels and then superimposed. Fig. 3 shows the power spectrum for the antenna pattern displayed in Fig. 2 for the $N=8$ azimuth channels, each one depicted with a different color. The effective PRF and the processed bandwidth intervals are delimited by the dashed black and red lines, respectively. For any arbitrary average rate $\bar{R}$, the set of quantization rates to be used for the transformed coefficients is entirely described by the array $\Delta \mathbf{R}=\left\{\Delta R_{k}\right\}$, defined as in (3). For the system configuration as in Fig. 3 it results $\Delta \mathbf{R}=[-2.0,-1.3,+0.9,+0.8,+0.9,+0.8,+1.0,-1.1]$ bits per sample, i.e. the three (high-Doppler) channels depicted in black, blue and brown in Fig. 3, respectively, will be quantized with less bits than the average rate $\bar{R}$, whereas, for the five channels lying in the center of the Doppler spectrum, about one bit/sample more will be allocated.

To assess the performance of the proposed MC-BAQ method we have evaluated the signal-to-quantization noise ratio (SQNR) on the focused SAR image, defined as the power ratio of the non-compressed signal $s$ to the quantization error $q=s-\hat{s}$ affecting the reconstructed signal $\hat{s}$

$$
\text { SQNR }=\frac{\sum_{p=1}^{P}\left|s_{p}\right|^{2}}{\sum_{p=1}^{P}\left|q_{p}\right|^{2}},
$$

being $P$ the total number of pixels. As an example, Fig. 4 shows the SQNR obtained for a homogeneous scene, as a function of the average rate $\bar{R}$, for the system parameters in Table I and for different quantization schemes: the performance of a standard BAQ is taken as reference and is depicted in red. Then, the SQNR for the proposed MC-BAQ with the bit allocation derived from rate distortion theory (R-D) as in (3) is shown in turquoise: as an example, a 4-bit BAQ has the same SQNR as a 3.5-bit MC-BAQ (both around $20 \mathrm{~dB}$ ), hence allowing for a saving of about 0.5 bits/sample. However, we could verify that the rate sequences derived as in (3) do not actually correspond to the optimum ones, i.e. there exist other sets of quantization rates that lead to better performance. As already pointed out, a reason for this could 


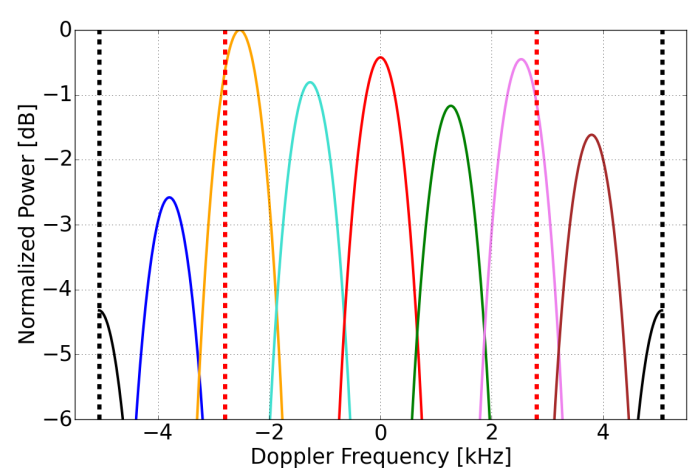

Fig. 3. Doppler power spectrum for the $N=8$ azimuth channels, depicted with different colors, each one scaled by the corresponding power contribution $\mathbf{P}_{k}$, as in (5). The power associated to each channel $\sigma^{2}{ }_{k}$ is estimated by integrating the corresponding power spectrum in the range between $-\mathrm{PBW} / 2$ and PBW/2 (indicated by the dashed red lines), according to (4). The dashed black lines delimit the PRF interval.

be that R-D theory (from which (3) is derived) is strictly valid for high-rates approximation hypothesis, which is not the case for the typical bit rates considered in this scenario. Moreover, the SQNR associated to the typically fractional bit rate $R_{k}$ resulting from (3) is a nonlinear function of the SQNR values associated to the integer BAQ rates used for its implementation according to [12], which further impacts the final performance. Hence, for the given system PRF, PBW, and antenna patterns, the optimum rate sequence has been derived by iterative search. For this, we used as initial point the values obtained from R-D theory, and then searched for the set of quantization rates which provides the best SQNR. The result is shown by the green curve and dots in Fig. 4. Again, if considering as target performance the one obtained with a 4-bit BAQ, with the proposed MC-BAQ one would get a gain of about $4 \mathrm{~dB}$ with respect to a standard BAQ operating at the same rate. Alternatively, the same SQNR is shown by the MC-BAQ at 3.25 bits/sample, hence achieving a data reduction of about $18-20 \%$. On the other hand, if a 3-bit BAQ is used as reference, the resulting data reduction is around $25 \%$. An example of the optimization procedure for the MC-BAQ bit rate allocation described above (i.e. the green line in Fig. 4) is shown in Fig. 5, which depicts the SQNR as a function of different combinations of quantization rate sets. Based on the symmetry shown by the power channels distribution in Fig. 3, we can describe, with reasonable approximation, any possible rate sequence by means of a set of three bit rate values $\left\{R_{\text {High }}, R_{M i d}, R_{\text {Low }}\right\}: R_{\text {High }}$ (the largest rate) is associated to the five sub-bands located in the center of the Doppler spectrum of Fig. 3, $R_{M i d}$ refers to the bit rate for the two adjacent channels (blue and brown sub-bands in Fig. 3), and $R_{L o w}$ is for the black sub-band in Fig. 3. The latter represents the lowest rate and, once defined the average rate $\bar{R}$, it is uniquely determined as $R_{\text {Low }}=\bar{R}-5 \cdot R_{\text {High }}-2 \cdot R_{\text {Mid }}$. According to this, the best SQNR, of about $23.5 \mathrm{~dB}$, is achieved for $\left\{R_{\text {High }}, R_{\text {Mid }}, R_{\text {Low }}\right\}=\{5.6,2.0,0.0\}$ bits/sample, as indicated by the dashed black lines sketched in Fig. 5. Clearly, setting $R_{\text {Low }}=0$ means that the corresponding DFT coefficient is just discarded on board and hence not downlinked at all.

In addition, we have assessed the proposed method for the

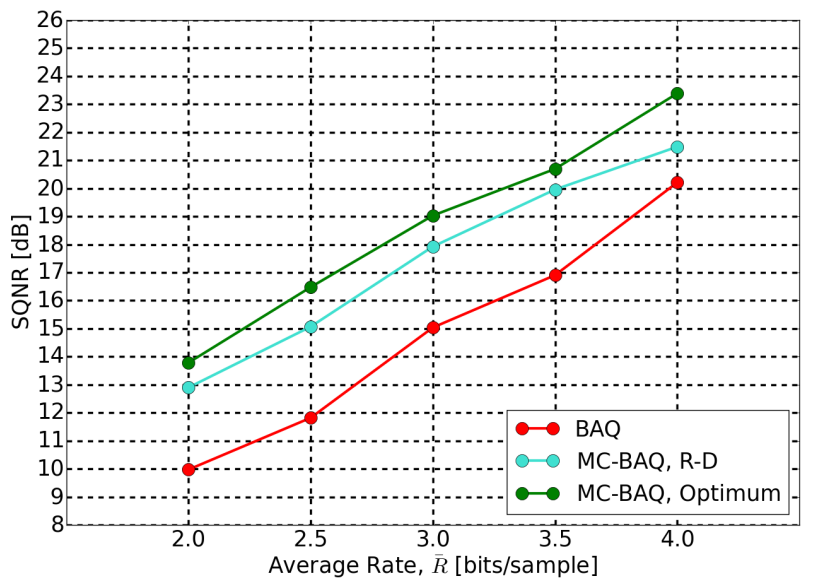

Fig. 4. Signal-to-quantization noise ratio (SQNR) for a homogeneous scene for different quantization schemes: standard BAQ (red), MC-BAQ with bit allocation derived from rate distortion theory (R-D, turquoise), and MC-BAQ with optimum bit allocation (green), as a function of the average rate $\bar{R}$.

backscatter profile depicted in Fig. 6 in black, which shows a "jump" of $10 \mathrm{~dB}$ (left vertical axis) along azimuth, typically occurring over highly inhomogeneous targets, such as, e.g., urban areas. The resulting SQNR profiles (right vertical axis) are shown for a standard BAQ in red and for the proposed MC-BAQ in green (for this, we have selected the optimum bit allocation as in Fig. 5), and a consistent performance gain can be observed. In particular, a significant loss in terms of SQNR is observed for both compression techniques in the part of the low backscatter area (with a mean backscatter of -10 $\mathrm{dB})$ close to the high backscatter one. This is due the masking effect affecting SAR data quantization, which is induced by the presence of high backscatter targets within a distance comparable to the synthetic aperture $L_{s a}$ [14], [15] (for the considered system, $L_{s a}$ is in the order of a few tens of kilometers). As already pointed out, the performance of the proposed method strongly depends on the ratio $r=\mathrm{PBW} / \mathrm{PRF}_{\text {eff }}$, i.e. the closer $r$ is to one, the lower the resulting gain. Different values of $r$ have been considered by varying the processed bandwidth only. As an example, for $r=0.6$ the data rate can be reduced by about 0.5 bits/sample, whereas for $r=0.7$ only a negligible performance gain is obtained, making the proposed method not suitable for data reduction purposes. On the other hand, the number of azimuth receive channels $N$ also plays a key role in determining the achievable performance of MC-BAQ: the larger $N$ is, the better the power spectrum of the transformed coefficients (shown in Fig. 3 for $N=8$ ) approximates the actual one, hence more accurate bit allocation and ultimately more effective data reduction can be achieved. Regarding the required onboard complexity, the proposed multi-channel compression technique can be performed in real time by using a state-of-the-art FPGA without excessive memory storage and retrieval capability. For the implementation of a $N$ points FFT (being $N$ the number of azimuth channels) a limited amount, in the order of a few tens, of additional operations $N_{o p}$ is required for each received range line and multi-channel azimuth block, being $N_{o p}=\mathcal{O}\left(N \log _{2} N\right)$. 


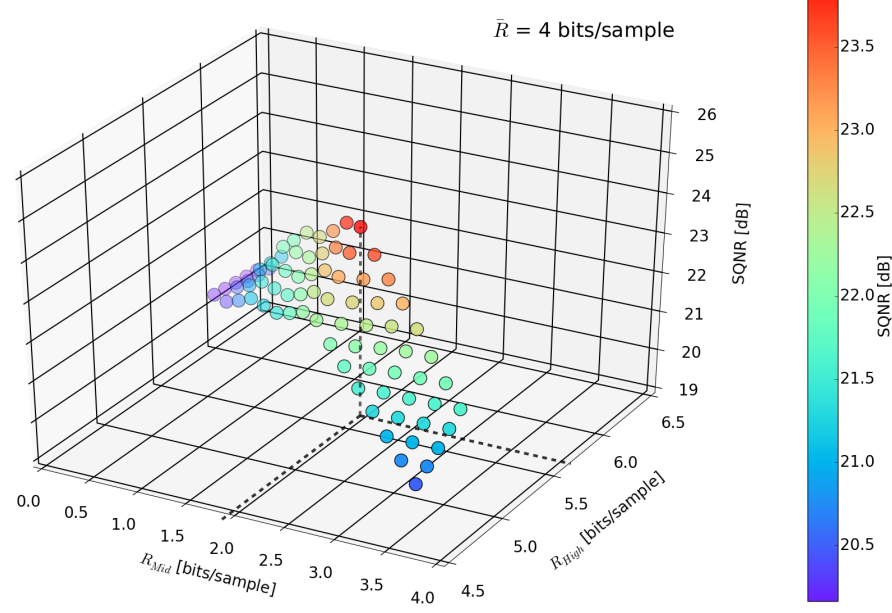

Fig. 5. SQNR for different combinations of quantization rates applied for the proposed MC-BAQ for $\bar{R}=4$ bits/sample. Based on the symmetry shown by the power channels distribution in Fig. 3, we approximate any sequence by a set of three bit rates $\left\{R_{H i g h}, R_{M i d}, R_{L o w}\right\}$, each one associated to different Doppler sub-bands of Fig. 3. The dashed black lines indicate the maximum SQNR, of about $23.5 \mathrm{~dB}$, which is achieved by setting $R_{H i g h}=5.6$ bits/sample and $R_{M i d}=2$ bits/sample.

\section{CONCLUSION}

In this letter we propose a novel method for onboard data volume reduction for multi-channel SAR systems. Such systems require the acquisition of an increased volume of data in order to achieve high-resolution imaging of a wide swath width. The suggested approach exploits the intrinsic correlation exhibited by the received multi-channel SAR raw data samples. For this purpose, a discrete Fourier transform combined with an optimized selection of the quantization rates is applied to the multi-channel azimuth data samples. Simulations are carried out for a single-platform C-band system with eight azimuth receive channels, and for different SAR backscatter distributions, showing that with the proposed method a data volume reduction of $20 \%-25 \%$ can be achieved for typical BAQ rates employed for SAR applications. Future studies will include the investigation of alternative orthogonal transformations and the detailed analysis of different system configurations, in terms of, e.g., antenna patterns, number of azimuth channels, and required processed bandwidth and PRF. MC-BAQ can also be exploited in the context of polarimetric SAR data, by applying it on each polarization channel independently. Since the power spectrum of the azimuth antenna pattern is approximately the same for all polarization channels, one could practically employ the same set of bit rates $\left\{R_{k}\right\}$ for each of the available polarimetric combinations. Finally, the proposed method could be extended to multiple transmit pulses by combining transform coding in the Doppler domain with alternative compression schemes such as, e.g., vector quantization [9], to achieve a more effective data reduction.

\section{ACKNOWLEDGMENT}

This research was partly funded by the European Space Agency (ESA/ESTEC) project "Calibration and data reduction for digital beamforming instruments" Contract Nr. 4000117363/16/NL/FE.

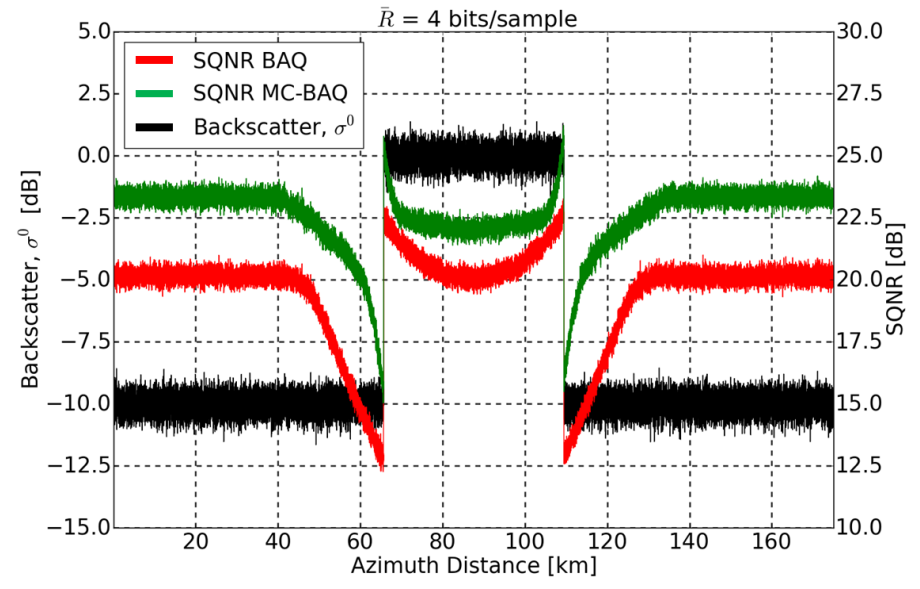

Fig. 6. Backscatter profile $\left(\sigma^{0}\right.$, in black) and corresponding SQNR as a function of the azimuth distance for BAQ (red) and MC-BAQ (green) for an average rate $\bar{R}$ of 4 bits/sample. The performance gain obtained with the proposed method can be exploited to reduce the resulting data rate.

\section{REFERENCES}

[1] G. Krieger, N. Gebert, and A. Moreira, "Unambiguous SAR signal reconstruction from non-uniform displaced phase center sampling," IEEE Geosci. and Remote Sens. Lett., vol. 1, no. 4, pp. 260-264, October 2004.

[2] N. Gebert, G. Krieger, and A. Moreira, "Digital beamforming on receive: Techniques and optimization strategies for high-resolution wide-swath SAR imaging," IEEE Trans. Aerosp. Electron. Syst., vol. 45, no. 2, pp. 564-592, April 2009.

[3] M. Villano, G. Krieger, and A. Moreira, "Onboard processing for data volume reduction in high-resolution wide-swath SAR," IEEE Geosci. Remote Sens. Lett., vol. 13, no. 8, pp. 1173-1177, August 2016.

[4] P. Guccione, M. Scagliola, and D. Giudici, "Principal components dynamic block quantization for multichannel SAR," in IEEE Int. Geosci. Remote Sens. Symp., Bejing, China, July 2016, pp. 2090-2093.

[5] M. Martone, M. Villano, M. Younis, and G. Krieger, "An efficient onboard quantization strategy for multi-channel SAR systems," in Proc. EUSAR, Aachen, Germany, June 2018, pp. 1-6.

[6] D. Cerutti-Maori, I. Sikaneta, J. Klare, and C. H. Gierull, "MIMO SAR processing for multichannel high-resolution wide-swath radars," IEEE Trans. Geosci. and Remote Sens., vol. 52, no. 8, pp. 5034-5055, August 2014.

[7] F. Q. de Almeida, M. Younis, G. Krieger, and A. Moreira, "Multichannel staggered SAR azimuth processing," IEEE Trans. Geosci. and Remote Sens., vol. 56, no. 5, pp. 2772-2788, February 2015.

[8] S. Barbarossa, "Detection and imaging of moving objects with synthetic aperture radar. Part 1. Optimal detection and parameter estimation theory." IEE Proceedings F - Radar Signal Processing, vol. 139, no. 1, pp. 79-88, January 1992.

[9] U. Benz, K. Strodl, and A. Moreira, "A comparison of several algorithms for SAR raw data compression," IEEE Trans. Geosci. and Remote Sens., vol. 33, no. 5, pp. 1266-1276, September 1995.

[10] R. Kwok and W. T. K. Johnson, "Block adaptive quantization of Magellan SAR data," IEEE Trans. on Geosci. and Remote Sens., vol. 27, no. 4, pp. 375-383, July 1989.

[11] N. S. Jayant and P. Noll, Digital Coding of Waveforms. Principle and Applications to speech and video. Prentice Hall, 1984.

[12] M. Martone, B. Bräutigam, and G. Krieger, "Azimuth-Switched Quantization for SAR systems and performance analysis on TanDEM-X data," IEEE Geosci. Remote Sens. Lett., vol. 11, no. 1, pp. 181-185, January 2014.

[13] M. Villano, G. Krieger, and A. Moreira, "Advanced spaceborne SAR systems with planar antenna," in IEEE Radar Conference, Seattle, WA, USA, May 2017, pp. 152-156.

[14] M. Martone, B. Bräutigam, and G. Krieger, "Quantization effects in TanDEM-X data," IEEE Trans. Geosci. and Remote Sens., vol. 53, no. 2, pp. 583-597, February 2015.

[15] S. Huber, M. Younis, and G. Krieger, "The TanDEM-X mission: overview and interferometric performance," Int. J. Microw. Wireless Technol., vol. 2, no. 3-4, pp. 379-389, July 2010. 